ISBN 978-81-933894-6-1

7th International Conference on Recent Trends in Science, Engineering and Technology

(RTSET-2017)

London (U K) June 29-30, 2017

\title{
First Stage Cooperative Network Coding for Wireless Sensor Network
}

\author{
Dr. Hani Attar \\ Philadelphia University, Jordan \\ Attar_hani@hotmail.com
}

\begin{abstract}
Network Coding (NC) and cooperation network are two well-joint technologies, as NC tents to combine data for different transmitters, so, each transmitter help in transmitting other nodes in the network, which is what cooperative technique does so, i., e., Cooperative NC (CoNC) is defined as combining different users' data in a way that users help each others to transmit and receive their data.The proposed work is applying CoNC in the first stage in a deterministic manor to gain some benefits from this useful technique. Applying CoNC over the first stage requires sending the packets in two halves rather than one full half where CoNC is applied in the second half. The proposed CoNC protocol is applied over Wireless Sensor Network (WSN), resulting to better bandwidth and less power consumption with better Packet Error Probability for the proposed WSN. The proposed scenario is for $N$ nodes of WSN cluster that exchange data over an erasure channel.The proposed protocol provides the ability for the network to lose one packet between each couple of users ( $N$ packets over the whole network) in the first stage, resulting to decrease the Automatic Request significantly in the first stage.
\end{abstract}

\section{Introduction}

Network Coding (NC) is such old technique that was proposed in [1] over wireline network in a multicasting information scenario, resulting to a significant improvement in the data rate and power consumption, mainly because NC technique tends to transmit less number of combined packets through the uplink channel rather than to transmit the large original number of packets separately (uncombined).

Network Cooperation is a technique where a user cooperates with other users to improve the diversity, the channel bandwidth and the power consumption by transmitting less packets over the network and decreasing the number of Automatic Repeat Request (ARQ) for the lost packets over the network. Accordingly, when the network joins the principle of NC and Cooperation; it is called Cooperative NC (CoNC) network [2] and [3].

In the wireless communication, $\mathrm{NC}$ and $\mathrm{CoNC}$ are widely used mainly because of the broadcasting natural of the network, where all users in the network can receive all the other user's packets and hence, apply NC or/and CoNC before forwarding them to the rest of users in the same network [2] and [3].

In [4], CoNC was applied over a network with a BS, where NC was applied cooperatively in a single BS resulting to such good improvement in the Packet Error Rate (PER) beside the better ARQ, because the network has good tolerance to lose a large number of the transmitted packets over the investigated erasure channel, with the guarantee of the full connectivity.

In [2] and [5], CoNC was applied over WSN over Rayleigh Fading channel, represented by Finite State Markov Chain (FSMC) providing an acceptable practical results for using CoNC over WSN when a certain number of users are placed in equal distance for a destination.

In [6], CoNC was applied over an erasure channel for the application of Long Term Evaluation mobile network, and it shows how the network performance improved significantly mainly in term of power consumption and data rate. 
In [7], [8], and [9], NC was investigated over the physical layer in good details with different forward error correction codes and deferent scenarios to prove that $\mathrm{NC}$ and/or CoNC are practical bandwidth technique that has such acceptable BER over the physical layer.

In [10] two senders communicate through one relay with two receivers in a full duplex channel model was proposed and provided good understanding for NC behavior.

The recent work in [11] provides a useful coding algorithm that fits the dynamical network by taking the advantage of the feedback three-receiver scenario.

The feedback advantage for WSN has been recently investigated in [12], for a practical blind instant decoding network over a lossy feedback environment. It is important to assure that it is possible to apply the feedback scenario in the proposed work in this paper, to even gain more bandwidth saving and power consumption.

In [13], [14], and [15], most recent work applied over WSN which has applied CoNC with different important techniques and we believe it gives good and important knowledge for the most updated CoNC WSN techniques which are unlike the proposed work in this paper mainly because our proposed CoNC starts in the first stage and being applied in each half stage rathe $r$ than each full stage.

The rest of the paper is organized as following: Section 2 explains the system model and Section 3 introduces the proposed system model, followed by Section 4 which shows the proposed protocol for WSN based on CoNC. The simulation results are shown in Section 5 to show the benefits that are gained by applying the proposed technique. Finally, Section 6 concludes the paper.

\section{System Model}

In this paper, we investigate a WSN that allows $\mathrm{N}$ nodes to exchange their data over erasure channels between them all, where $\mathrm{N} \geq 2$ nodes $\mathrm{N}_{1}, \mathrm{~N}_{2}, \ldots, \mathrm{N}_{\mathrm{N}}$.

In the traditional way of communication "benchmark scenario" where neither NC nor Cooperation is applied, each user sends its own packet alone.

Figure 1: WSN example for the first stage when $N=4$ Nodes where $N_{i}$ broadcasts $X_{i}$ to $N_{j}$ nodes $\left(X_{i-j}\right)$ and $i \neq j$. The probability of not receiving the packet between any two users is $q$ (the erasure channel probability), for simplicity and without losing the generality, we assume that $\mathrm{q}$ is the same for all channels and each node informs the N-1 neighbors with decoding all packets successfully by broadcasting a simple feedback message and this message is assumed to be guaranteed transmitted.

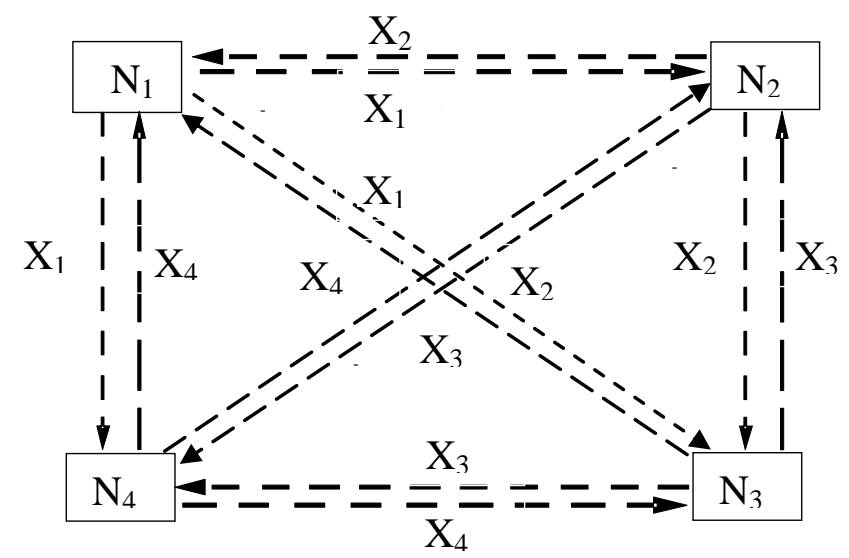

Fig. 1 Shows the WSN Nodes for Four Nodes (N=4) For the First Stage in the Benchmark Scenario

The PER for full reception at each node and all nodes after the first and second stage when CoNC is not applied, and when it is applied after the first stage is shown in [4].

So, at the end of the first stage, each node will be receiving a maximum of $\mathrm{N}-1$ nodes, taking into consideration that each node knows its own packet. The same for the end of the second stage, where each node 
will be receiving a maximum of $2(\mathrm{~N}-1)$ packets, i. e., the same of $\mathrm{N}-1$ packets twice if all packets are successfully received.

The proposed protocol in this paper is mainly directed to solve the benchmark scenario disadvantages, resulting to better bandwidth, power consumption and PER network, which is in introduced in the following section.

\section{2- Proposes CoNC system:}

The proposed CoNC is applied over the first in term of half-stage transmission.

\subsection{First Stage Proposed CoNC System Model}

We propose a simple and practical protocol that tends to apply CoNC in the first stage to not miss the benefit that can be obtained from this useful technique, taking into consideration that the proposed protocol does transmit the same number of packets $\mathrm{N}$ packet after the first stage.

The proposed combination strategy is regarded as a simple XORing addition resulting to the same information length for the combined packet.

Indeed, if the $\mathrm{N}$ sensor cluster nodes are divided to two sub-clusters, where each cluster contains N/2 nodes, and the sub-cluster sensor nodes transmit their packet as a separate stage, we can assume that each sub-cluster is regarded as a half-stage and the communication will be performed in term of half-stages instead of full ones.

To simplify the protocol, we assume that the odd node numbers consist the first sub-cluster while the rest (even numbers) nodes consist the second sub-cluster where odd or even number of users will not change the proposed protocol algorithm.

First of all, the odd nodes (odd sub-cluster) transmit their packets, where the even nodes (even sub-cluster) are remaining in the receiving mode. Accordingly, the first half-stage transmission ends while each node in the whole network knows all the odd sub-cluster nodes in the case of full reception, and no any CoNC is applied in the first half-stage.

At the end of the first half-stage, each node will be receiving odd sup-cluster nodes.

In the second half-stage, the even sup-cluster nodes will be in transmission mode, while the odd sub-cluster nodes will be in receiving mode.

The even sup-cluster nodes will apply CoNC in this half-stage instead of sending their separate nodes alone.

In the second half-stage; each even node will add all the received odd node packets to their own packet, ending up with sending N/2 packets in the second half-stage as well.

Total of transmitted packets after the first stage remains the same as in full one stage scenario which is $\mathrm{N}$. An example of six sensor nodes $(\mathrm{N}=6)$ is shown in figure 2,

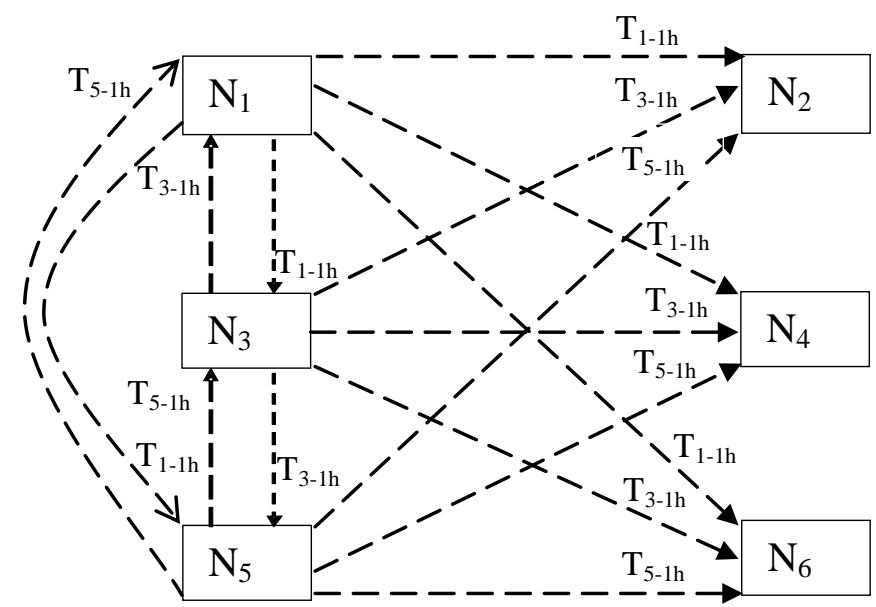

Fig. 2: First half-stage transmission where CoNC is not applied.

Where $\mathrm{T}_{\mathrm{i}-1 \mathrm{~h}}$ is the transmitted packet from node $\mathrm{i}$ at the first half-stage while $\mathrm{i}=1,2, \ldots, \mathrm{N}$.

It is clear that each node will know all the N/2 odd packets in the case of full reception as shown in Eq. (1): 
$\left[\begin{array}{l}T_{1-1 h} \\ T_{2-1 h} \\ T_{3-1 h} \\ T_{4-1 h} \\ T_{5-1 h} \\ T_{6-1 h}\end{array}\right]=\left[\begin{array}{llllll}X_{1} & X_{2} & X_{3} & X_{4} & X_{5} & X_{6}\end{array}\right]\left[\begin{array}{llllll}1 & 0 & 0 & 0 & 0 & 0 \\ 0 & 0 & 0 & 0 & 0 & 0 \\ 0 & 0 & 1 & 0 & 0 & 0 \\ 0 & 0 & 0 & 0 & 0 & 0 \\ 0 & 0 & 0 & 0 & 1 & 0 \\ 0 & 0 & 0 & 0 & 0 & 0\end{array}\right]$

where $\mathrm{X}_{\mathrm{i}}$ is the transmitted packet from node $\mathrm{i}$ where $\mathrm{i}=1,2, \ldots, \mathrm{N}$.

From Eq. (1), we notice that just the odd nodes transmit their own packets separately and alone, resulting to Eqs. (2), (3), (4) and (5):

$T_{1-1 h}=X_{1}$

$T_{3-1 h}=X_{3}$

$T_{5-1 h}=X_{5}$

$T_{2-1 h}=T_{4-1 h}=T_{6-1 h}=0$

The transmitted packets by the even nodes are given in Eq. (6)

$\left[\begin{array}{l}T_{1-2 h} \\ T_{2-2 h} \\ T_{3-2 h} \\ T_{4-2 h} \\ T_{5-2 h} \\ T_{6-2 h}\end{array}\right]=\left[\begin{array}{llllll}X_{1} & X_{2} & X_{3} & X_{4} & X_{5} & X_{6}\end{array}\right]\left[\begin{array}{llllll}0 & 0 & 0 & 0 & 0 & 0 \\ 1 & 1 & 1 & 0 & 1 & 0 \\ 0 & 0 & 0 & 0 & 0 & 0 \\ 1 & 0 & 1 & 1 & 1 & 0 \\ 0 & 0 & 0 & 0 & 0 & 0 \\ 1 & 0 & 1 & 0 & 1 & 1\end{array}\right]$.

Where $T_{i-2 h}$ is the transmitted packet from node $i$ in the second half-stage while $i=1,2, \ldots, N$. From Eq. (6); the even sup-cluster nodes apply CoNC by transmitting their own packets combined with the packets received in the first half-stage.

Figure 3 shows the second half-stage

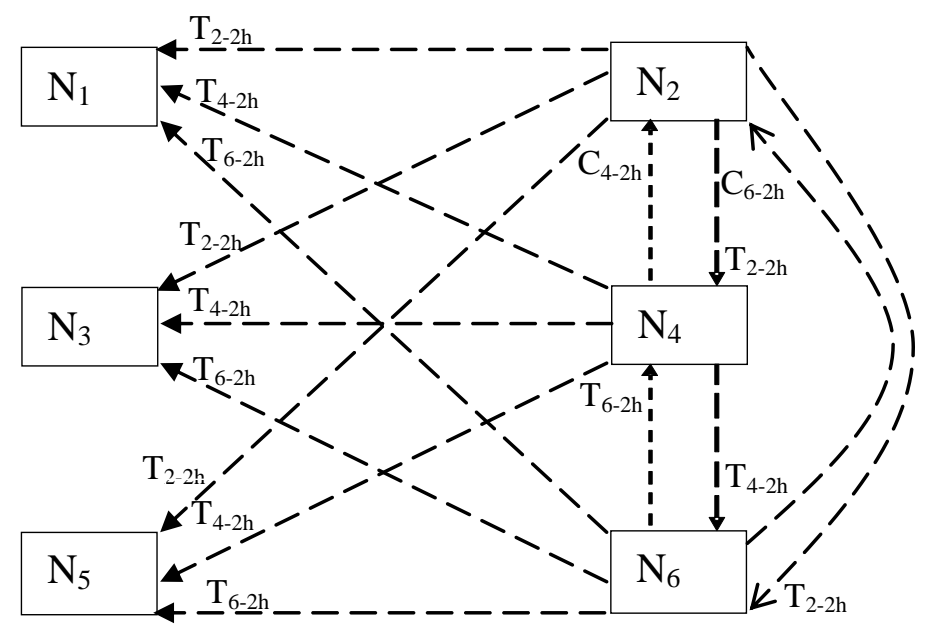

Fig. 3: The transmitted packets by the even sup-cluster nodes after applying CoNC.

In figure 3, each node in the even sup-cluster broadcasts its own packet combined with the odd packets received in the first half-stage, Accordingly,

$T_{2-2 h}=X_{2} \oplus X_{1} \oplus X_{3} \oplus X_{5}$

$T_{4-2 h}=X_{4} \oplus X_{1} \oplus X_{3} \oplus X_{5}$

$T_{6-2 h}=X_{6} \oplus X_{1} \oplus X_{3} \oplus X_{5}$

$T_{1-2 h}=T_{3-2 h}=T_{5-2 h}=0$

At the end of the first stage, i. e., firs odd sup-cluster and second even sup-cluster stages, each user will be able to have $T_{i-j h}$ different packets where $i=1,2, \ldots, N$ and $j=1,2$, taking into consideration that $\mathrm{N}_{i}$ node replaces its own packet $\left(X_{i}\right)$ with $T_{i-j h}$ where $j=1,2$, and $i=j$, for an example, node 2 replaces $T_{2-2 h}$ with $X_{2}$ at its end.

In conclusion, each user receives five different packets and knows its own at the end of the first stage as shown in Eq. (11) at the forth $\left(\mathrm{N}_{4}\right)$ node for example: 


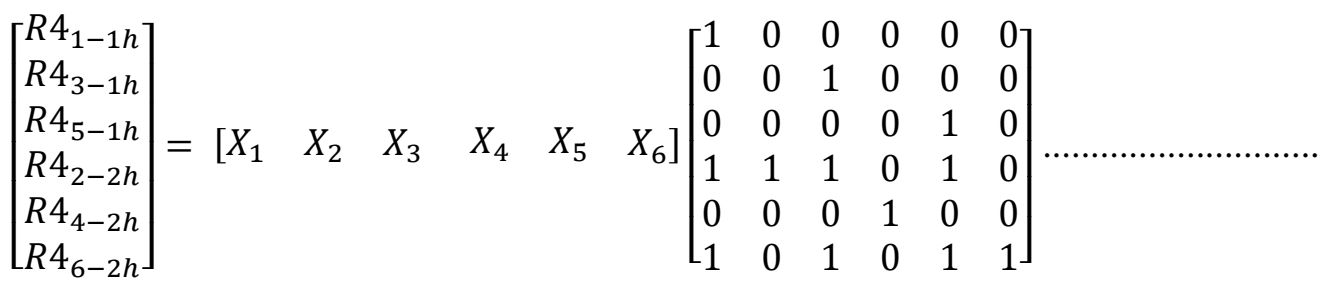

Where $\mathrm{R} 4_{\mathrm{i}-\mathrm{jh}}$ is the received packet at node 4 from node $\mathrm{i}$ in $\mathrm{j}$ half-stage where $\mathrm{i}=1,2, \ldots, \mathrm{N}$ and $\mathrm{j}=1,2$. For Eq. (11), we find out that $R 4_{4-2 h}$ is equation to $\mathrm{X}_{4}$

When comparing Eq. (11) with traditional first stage transmission where CoNC is not applied, we can conclude the following gained advantage:

Theory 1: The $\mathrm{N}$ transmitted packets after the first stage give $\mathrm{N}$ linearly independent equations with rank $\mathrm{N}$ receiving matrix.

Theory 3: The combination adapted in the second half-stage is a deterministic combination, which simplify the GJE significantly [3], Eq. (11) shows this clearly.

Theory 4: If any packet transmitted from the second half-stage is not received at any node in the whole network, the system will not be able to decode its node packet as the remaining rank of the received matrix is less than $\mathrm{N}$.

\section{Simulation Results}

The proposed scenario is simulated using Matlab program, where different number of WSN cluster size is simulated.

The program runs till collecting a minimum of 100 errors, where the error is identified as the failure of any user $i$ to receive any packet from the N-1 member in the cluster. So, we are seeking full connectivity between the $\mathrm{N}$ cluster nodes. Moreover, the results are collected at node 4, and hence, seeking to receive the other N-1 packets.

The collected results for the proposed combining protocol are compared with the benchmark results, where CoNC is not applied, which is the case where each node just sends its own packet separately.

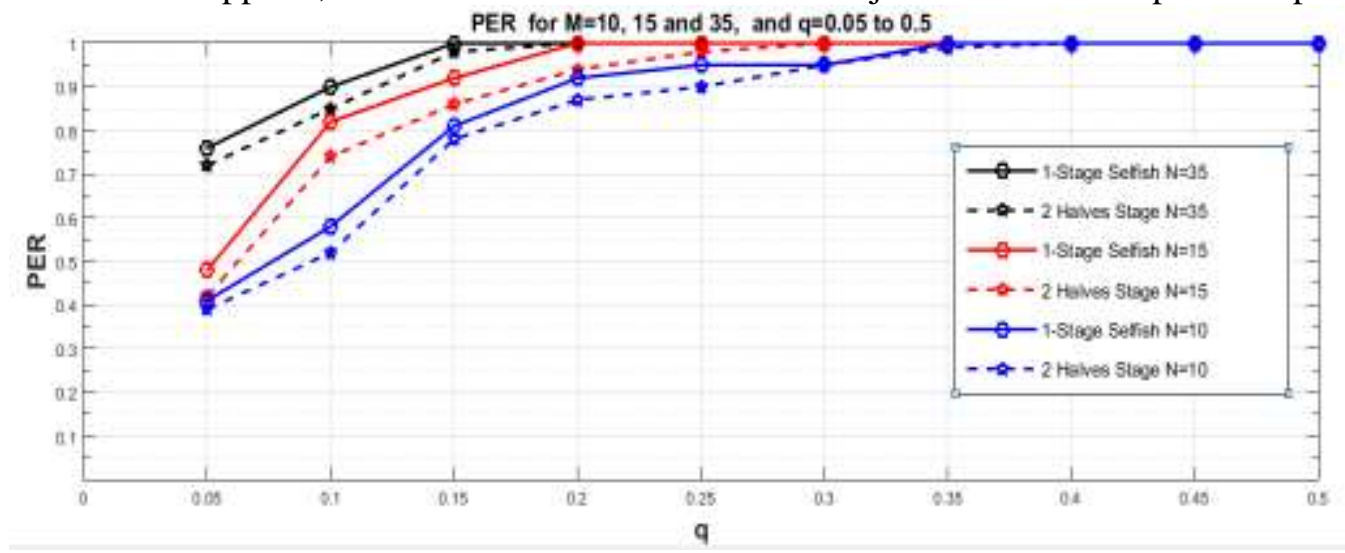

Fig. 4: PER for single stage selfish system compared with the proposed two halves stage.

Fig. 4 shows that the proposed protocol improves the PER by almost 10\%, which confirms the theoretical point of view.

Moreover, it is clear that the when the cluster size increases, the full connectivity becomes more and more difficult, in the benchmark scenario, and in the proposed two halves scenario, however, it is clear that the proposed protocol does maintain the $10 \%$ improvement even for large num number of nodes.

More importantly, the results show that applying the transmission in half stages gives us the opportunity to apply $\mathrm{CoNC}$ in the first stage and a half stage scenario, which is the main propose to prove in this work.

\section{Conclusion}

Network Coding (NC) is usually applied over the second stage transmission as in the first stage, all users receive others' data, and hence start applying NC together with Cooperation technique in the second stage, however, in this work, the Cooperative $\mathrm{NC}(\mathrm{CoNC})$ was applied in the first stage and gained good performance 
improvement in term the ability to network to drop till $10 \%$ of the transmitted packets without the need to result to the Automatic Repeat Request (ARQ), which results to save the transmission power and increases the network reliability.

The results prove that the idea of half stage transmission is applicable and it leads to save in the number of the transmitted packets resulting to obtain better bandwidth and less transmission traffic.

Future work will be directed to go for extra halves stage in the case of not full reception declared after the first stage (first two halves), which enables the system save the required extra packets to insure the full connectivity. In future work, author is planning to apply CoNC over the routing model published in [16] and [17] to investigate the advantage that can be gained.

\section{References}

[1] R. Ahlswede, N. Cai, S.-Y.R. Li, and R.W. Yeung, "Network information flow," IEEE Trans. Inform. Theory, vol. 46, pp. 1204-1216, July 2000. https://doi.org/10.1109/18.850663

[2] H. Attar, D. Vukobratovic, L. Stankovic and V. Stankovic, "Performance Analysis of Node Cooperation with Network Coding in Wireless Sensor Networks," 2011 4th IFIP International Conference on New Technologies, Mobility and Security, Paris, 2011, pp. 1-4. doi: 10.1109/NTMS.2011.5721048 https://doi.org/10.1109/NTMS.2011.5721048

[3] Attar, Hani, Stankovic, Lina and Stankovic, Vladimir (2012) Cooperative network-coding system for wireless sensor networks. IET Communications, 6 (3). pp. 344-352. ISSN 1751-8628 https://doi.org/10.1049/iet-com.2011.0143

[4] Mohamed El-Hihi1, Hani Attar1, Ahmed A. A. Solyman, Lina Stankovic, " Network Coding Cooperation Performance Analysis in Wireless Network over a Lossy Channel, M Users and a Destination Scenario" Vol.8 No.4, Nov 2016.

[5] Alhihi, M. (2017) Network Coding for Wireless Sensor Network Cluster over Rayleigh Fading Channel: Finite State Markov Chain. Int. J. Communications, Network and System Sciences, 10, 1-11. http://dx.doi.org/10.4236/ijcns.2017.101001

[6] H. Attar, L. Stankovic, M. Alhihi and A. Ameen, "Deterministic network coding over Long Term Evaluation Advance communication system," 2014 Fourth International Conference on Digital Information and Communication Technology and its Applications (DICTAP), Bangkok, 2014, pp.56-61.

doi: 10.1109/DICTAP.2014.6821657

https://doi.org/10.1109/DICTAP.2014.6821657

[7] Shengli Shang, "Applying physical-layer network coding in wireless networks", EURASIP Journal in Wireless Communications and Network, Ariticle No. 1 Volume Jan 2010.

[8] Soung Chang Liew, Lu Lu, and Shengli Zhang, " A primer on Physical Layer Network Coding" ISBN:9781627050920, DOI 10.2200/s00646ED1V01Y201505CNT016, Copyright 2015 by Mongan and Claypool.

[9] Attar, H. (2016) Physical Layer Deterministic Network Coding Using PUM Turbo Codes over AWGN Channel, N Nodes through a Base Station Scenario. Communications and Network, 8, 241-256. https://doi.org/10.4236/cn.2016.84022

[10] Sachin Katti, Ivana Maric Dina Katabi, Andrea Goldsmith and Muriel Medard "Joint Relaying and Network Coding in Wireless Networks"ISIT ,France, June, 2007.

[11]Douik, Ahmed S.; Sorour, Sameh; Al-Naffouri, Tareq Y.; Alouini, and Mohamed-Slim " Delay Reduction for Instantly Decodable Network Coding in Persistent Channels With Feedback Imperfections" 14 (11):5956 IEEE Transactions on Wireless Communications, 2015.

[12] S. Sorour, A. Douik, S. Valaee, T. Al-Naffouri, and M. Alouini, "Partially blind instantly decodable network codes for lossy feedback environment," IEEE Transactions on Wireless Communications, vol. 13, no. 9, pp. 4871-4883, Sept 2014 https://doi.org/10.1109/TWC.2014.2321397.

[13]O. T. Valle et al., "Experimental assessment of using network coding and cooperative diversity techniques in IEEE 802.15.4 wireless sensor networks," 2016 IEEE World Conference on Factory Communication Systems (WFCS), Aveiro, 2016, pp. 1-4. doi: 10.1109/WFCS.2016.7496521

[14] Odilson T. Valle, Carlos Montez, Gustavo Medeiros de Araujo, Francisco Vasques, and Ricardo Moraes " NetCoDer: A Retransmission Mechanism for WSNs Based on Cooperative Relays and Network Coding", Sensors 2016, ISSN:1424-8220, ESSN:1424-8220, Doi: 10.3390/s16060799, WW.mdpi.com/journal/sensors.

[15] R. R. Rout, S. K. Ghosh and S. Chakrabarti, "Co-operative routing for wireless sensor networks using network coding," in IET Wireless Sensor Systems, vol. 2, no. 2, pp. 75-85, June 2012. doi: 10.1049/iet-wss.2011.0069

[16] M Alhihi, H Attar, and A Skulynichev " Researching the Impact of Parameters of the Developed Routing Models on Network Performance", Studeis in Engineering and Teechnology 4(1), 61-69.

DOI: https://doi.org/10.11114/set.v4i1.2470

[17] Alhihi, Mohammad, "Practical Pouting Protocol Models to Improve Network Performance and Adequacy.' Journal of Computer and Communication 5.6 (217): 11 